\title{
Genomic and antigenic properties of Newcastle Disease virus genotypes 2.XX1 and 2.VII from Egypt do not point to antigenic drift as a driving force of spread
}

\author{
Mahmoud Naguib ${ }^{1}$, Dirk Höper ${ }^{2}$, Magdy El-Kady ${ }^{3}$, Manal Afify ${ }^{4}$, Ahmed Erfan ${ }^{5}$, \\ Hassanein Abozeid ${ }^{4}$, Wafaa Hassan ${ }^{5}$, Abdel-Satar A. Arafa ${ }^{5}$, Momtaz shahein ${ }^{5}$, Martin \\ Beer $^{2}$, Timm Harder ${ }^{2}$, and Christian Grund ${ }^{2}$ \\ ${ }^{1}$ Uppsala Universitet \\ ${ }^{2}$ Friedrich-Loeffler-Institute Federal Research Institute for Animal Health \\ ${ }^{3}$ Beni Suef University Faculty of Veterinary Medicine \\ ${ }^{4}$ Cairo University \\ ${ }^{5}$ Animal Health Research Institute
}

November 26, 2020

\begin{abstract}
Newcastle disease (ND), caused by avian orthoavulavirus type-1 (NDV), is endemic in poultry in the Middle East causing continuing outbreaks in poultry populations despite efforts to vaccinate. In the past, genotype 2.XXI (former 2.VI) was present in poultry in Egypt but has been replaced by genotype 2.VII. We investigated whether virus evolution contributed to superseding, and focused on the antigenic sites within the Heamagglutinin-Neuramindase (HN) spike protein. Full length sequences of a NDV genotype 2.VII isolate currently circulating in Egypt was compared to a genotype 2.XXI isolate that was present as co-infection with vaccine type viruses (2.II) in an historical isolate of the year 2011. Amino acid differences in the HN glycoprotein for both 2.XXI and 2.VII viruses amounted to $11,7 \%$ and $11,9 \%$ compared to LaSota vaccine type. However, mutations within the globular head (aa 126-570), bearing relevant antigenic sites, were underrepresented (aa divergence of 8,8\% and 8,1\% compared to $22,4 \%$ and $25,6 \%$ within the fragment encompassing cytoplasmic tail, transmembrane part and stalk regions (aa 1-125) for genotypes 2.XXI and 2.VII, respectively. Nevertheless, reaction patterns of HN-specific monoclonal antibodies revealed differences between vaccine type viruses and genotype 2.XXI and 2.VII viruses for specific epitopes. Accordingly, compared to Egyptian vaccine type isolates and the LaSota vaccine reference strain, single aa substitutions in 6 of 10 described neutralizing epitopes were found within the attachment protein. However, the same alterations in neutralization sensitive epitopes were present in old genotype 2.XXI as well as in newly emerged genotype 2.VII isolates. In addition, isolates were indistinguishable by polyclonal chicken sera raised against different genotypes including vaccine viruses. These findings suggest, that factors other than antigenic differences within the HN-protein account for facilitating spread of genotype 2.VII while displacing genotype 2.XXI viruses in Egypt.
\end{abstract}

Genomic and antigenic properties of Newcastle Disease virus genotypes 2.XX1 and 2.VII from Egypt do not point to antigenic drift as a driving force of spread

(Running title: Absence of antigenic drift in Newcastle Disease virus)

Mahmoud M. Naguib ${ }^{1,2}$, Dirk Höper ${ }^{3}$, Magdy F. Elkady ${ }^{4}$, Manal A. Afifi ${ }^{5}$, Ahmed Erfan ${ }^{2}$, Hassanein H. Abozeid $^{5}$, Wafaa M. Hasan ${ }^{2}$, Abdel-Satar Arafa ${ }^{2}$, Momtaz Shahein $^{2}$, Martin Beer ${ }^{3}$, Timm C. Harder ${ }^{3}$, Christian Grund ${ }^{3, \#}$ 
${ }^{1}$ Zoonosis Science Center, Department of Medical Biochemistry and Microbiology, Uppsala University, 751 21 Uppsala, Sweden

${ }^{2}$ National Laboratory for Veterinary Quality Control on Poultry Production, Animal Health Research Institute, Agriculture research centre, Giza 12618, Egypt

${ }^{3}$ Friedrich-Loeffler-Institut, Federal Research Institute for Animal Health, Institute of Diagnostic Virology, 17493 Greifswald Insel-Riems, Germany

${ }^{4}$ Department of poultry disease, Faculty of Veterinary Medicine, Beni-Suef University, Beni-Suef 62511, Egypt

${ }^{5}$ Department of Poultry disease, Faculty of Veterinary Medicine, Cairo University, Giza 11221, Egypt

\# Corresponding author:

Christian Grund

OIE, FAO and National Reference Laboratory for Newcastle disease, Suedufer 10, 17493 Greifswald Riems, Germany

Christian.Grund@fli.de

Key words: Newcastle disease; Newcastle disease virus; genotypes; Egypt; evolution; antigen sites;

\section{Abstract}

Newcastle disease (ND), caused by avian orthoavulavirus type-1 (NDV), is endemic in poultry in the Middle East causing continuing outbreaks in poultry populations despite efforts to vaccinate. In the past, genotype 2.XXI (former 2.VI) was present in poultry in Egypt but has been replaced by genotype 2.VII. We investigated whether virus evolution contributed to superseding, and focused on the antigenic sites within the Heamagglutinin-Neuramindase (HN) spike protein. Full length sequences of a NDV genotype 2.VII isolate currently circulating in Egypt was compared to a genotype 2.XXI isolate that was present as co-infection with vaccine type viruses (2.II) in an historical isolate of the year 2011. Amino acid differences in the HN glycoprotein for both 2.XXI and 2.VII viruses amounted to $11,7 \%$ and $11,9 \%$ compared to LaSota vaccine type. However, mutations within the globular head (aa 126-570), bearing relevant antigenic sites, were underrepresented (aa divergence of $8,8 \%$ and $8,1 \%$ compared to $22,4 \%$ and $25,6 \%$ within the fragment encompassing cytoplasmic tail, transmembrane part and stalk regions (aa 1-125) for genotypes 2.XXI and 2.VII, respectively. Nevertheless, reaction patterns of $\mathrm{HN}$-specific monoclonal antibodies revealed differences between vaccine type viruses and genotype 2.XXI and 2.VII viruses for specific epitopes. Accordingly, compared to Egyptian vaccine type isolates and the LaSota vaccine reference strain, single aa substitutions in 6 of 10 described neutralizing epitopes were found within the attachment protein. However, the same alterations in neutralization sensitive epitopes were present in old genotype 2.XXI as well as in newly emerged genotype 2.VII isolates. In addition, isolates were indistinguishable by polyclonal chicken sera raised against different genotypes including vaccine viruses.

These findings suggest, that factors other than antigenic differences within the HN-protein account for facilitating spread of genotype 2.VII while displacing genotype 2.XXI viruses in Egypt.

\section{Key words}

Newcastle disease virus, Egypt, pathogenicity, antigenic variation, vaccine.

\section{Introduction}

Newcastle disease (ND) is a highly contagious disease with significant clinical impact and serious losses in poultry worldwide. The causative agent, Newcastle disease virus (NDV) or avian paramyxovirus type-1 (APMV-1) is a species within the genus Avian orthoavulavirus 1, a member of the family Paramyxoviridae(Rima et al., 2019). The genome is composed of a negative-sense, single-stranded RNA of approximately 
$15 \mathrm{~kb}$ coding for at least seven proteins, in the 3' to 5' order nucleocapsid protein (NP), phosphoprotein $(\mathrm{P}), \mathrm{V}$ protein $(\mathrm{V})$, matrix protein $(\mathrm{M})$, fusion protein $(\mathrm{F})$, hemagglutinin-neuraminidase $(\mathrm{HN})$ and large RNA-dependent polymerase protein (L) (Steward et al., 1993). The HN protein recognizes and attaches to sialic acid receptors on the surface of permissive cells and mediates the fusion activity of the F protein at the cell membrane for release of the nucleocapsid complex into the cytoplasm (Lamb \& Parks, 2007).

Based on its pathogenicity, NDV is categorized into four groups with increasing virulence from apathogenic, lentogenic, mesogenic and velogenic (Alexander, 2000). The molecular basis for NDV pathogenicity is associated with the amino acid sequence motif of the protease cleavage site of the fusion protein, and the abilities of specific cellular proteases to cleave this protein. Based on phylogenetic analysis, NDV can be distinguished in two distinct classes, 1 and 2, with one (1.I) and twenty one genotypes, respectively (2.I-2.XXI) (Dimitrov et al., 2019). Whereas the majority of class 1 viruses are avirulent and have their natural reservoir in aquatic wild birds (Kim et al., 2007), class 2 comprises virulent viruses that are responsible for the outbreaks in poultry and evolved over time(P. J. Miller et al., 2010). Phylogenetic evidence suggests that in the decades prior to the 1970s, genotypes 2.II, 2.III and 2.IV were predominant in North America, Asia and Europe, respectively. Only genotype 2.II comprises of lentogenic, mesogenic and velogenic pathotypes (Czegledi et al., 2002; Czegledi et al., 2003). and vaccines derived from lentogenic strains of this genotype (Seal et al., 1996). Genotype 2.VI viruses emerged in epizootics in the Middle East and Asia in the 1960s, while in the early 1970s genotype 2.V (most likely of South American origin) caused outbreaks in North America and in Europe (Herczeg et al., 2001; Wehmann et al., 2003). Later, genotype 2.VI viruses gave rise to strains adapted to pigeons, the so-called pigeon type paramyxovirus (PPMV-1) (D. J. Alexander et al., 1985). The new NDV nomenclature proposal (Dimitrov et al., 2019) placed subgroups 2.VIai and 2.VIaii, encompassing the early strains circulating in poultry (Chong et al., 2013), into the new genotypes 2.XX and 2.XXI whereas PPMV-1 is forming the "new" genotype 2.VI with several sub-genotypes. Most recently, several sub-lineages of genotype 2.VII and 2.VIII emerged in the Far East and spread to further geographic areas (Herczeg et al., 1999). Genotype 2.VII is currently predominant among velogenic NDV and can be further subdivided i.e. with sub-lineages 2.VII.1.1 encompassing viruses that emerged in the 1990s in the Far East, Europe and Asia, and the Middle East; while 2.VII.1.2 viruses evolved in China, and circulated in South and East Africa, as well as in Israel and Pakistan (Dimitrov et al., 2019).

In Egypt, ND is endemic and despite extensive routine vaccination programs implemented in the commercial poultry farm sectors, new cases occur continuously, posing a threat to the national poultry industry. In Egypt, ND was identified for the first time in 1948 (Daubney \& Mansy, 1948), and in the last few years NDV 2.II and 2.VII genotypes were reported from the country (Radwan et al., 2013). Also, co-infections with infectious bronchitis and avian influenza viruses were recorded in Egypt (Moharam et al., 2019; Naguib et al., 2017; Samy \& Naguib, 2018). This has raised concerns regarding the efficacy of ND vaccination programs applied in the country. It has been hypothesized that, due to accumulated mutations as seen previously with highly pathogenic avian influenza virus (Grund et al., 2011), new NDV antigenic variants have arisen, that have an advantage to spread within a vaccinated population. In consequence, viruses that supersede previous virus populations should have a gain in fitness, here, mutations within antigenic relevant sites. To test this hypothesis, four isolates representing genotype 2-II $(n=2)$, the old genotype 2.XXI (former 2.VIa; $n=1)$ and the currently circulating genotype 2.VII.1.1 in Egypt $(\mathrm{n}=1)$ were genetically and antigenically analyzed, with special focus on the viral glycoproteins, that are facilitating receptor mediated virus uptake and are the main target for protective immune responses.

\section{Material and Method}

\section{Sample collection and virus identification}

Samples (oropharyngeal and cloacal swabs, trachea or kidneys) were obtained from outbreaks of systemic disease in ten different commercial chicken farms in Egypt during 2011-2014 as shown in table 1. Samples were collected by the Reference Laboratory for Quality Control on Poultry production (RLQP)-Animal Health Research Institute (AHRI) and Beni-Suef University, Egypt, and shipped to the Friedrich-LoefflerInstitute (FLI), Germany. Identification of NDV was based on real-time reverse transcriptase polymerase 
chain reaction (RT-qPCR) using the Biorad CFX1000 Real-Time PCR system. Briefly, RNA was extracted from collected samples by QiaAmp viral RNA extraction kit (Qiagen, Hilden, Germany) as recommended by the manufacturer's instructions. NDV nucleic acid was detected by SuperScript@ III One-Step RT-PCR using primers and probe specific to the $\mathrm{M}$ gene (Wise et al., 2004). Positive samples were subjected to another RT-qPCR to differentiate lentogenic from velogenic viruses based on the sequence of fusion protein cleavage site (FPCS) using primers and probe specific for the M and F genes (Aldous et al., 2001; Moharam et al., 2019).

\section{Virus isolation}

Hepatocellular carcinoma epithelial cells (LMH) (Kawaguchi et al., 1987) were cultured with $2 \mu \mathrm{g}$ TCPK Trypsin $/ \mathrm{mL}$ in T25 culture flasks and infected with $0.5 \mathrm{~mL}$ of the NDV-positive samples. Cells were incubated at $37^{\circ} \mathrm{C}$ until giant cell formation was observed or for up to 7 days before initiating a second passage following a freeze/thaw cycle. Further confirmation using hemagglutination (HA) and hemagglutination inhibition (HI) tests were conducted on the supernatant culture medium (OIE, 2012). Isolated viruses were aliquoted and kept at $-80{ }^{\circ} \mathrm{C}$ until further use.

\section{Plaque purification}

Plaque assay was used to obtain purified virus clones for selected samples showing unequivocal results with pathotype-specific RT-qPCRs. Briefly, different virus dilutions were incubated on confluent LMH cell monolayers cultured in six-well plates overnight at $37^{\circ} \mathrm{C}$. Then, virus inoculum was removed, cell layers washed carefully twice with sterile PBS and overlaid with modified Eagle's medium containing $1.8 \%$ agar. Infected cells were incubated at $37{ }^{\circ} \mathrm{C}$ and $55 \%$ humidity for three days. Finally, selected plaques were picked and propagated on LMH cells as described above for further characterization.

\section{Genetic characterization and phylogenetic analysis}

Amplification of a 698 bps fragment spanning part of the NP and the F2 gene of NDV was performed using SuperScript III One-Step RT-PCR system (Invitrogen, Carlsbad, CA, USA) following published protocols (Aldous et al., 2003). Amplicons were size-separated by agarose electrophoresis, excised and purified from gel using the QIAquick Gel Extraction kit (Qiagen, Hilden, Germany). Purified PCR products were used directly for cycle sequencing reactions using BigDye Terminator v3.1 Cycle Sequencing Kit (Applied Biosystems, Foster City, CA, USA). The sequencing reaction products were purified using NucleoSeq kit (MachereyNagel, Düren, Germany) and sequenced on an ABI PRISMß 3100 Genetic Analyzer (Life Technologies, California, USA).

Assembly of the obtained sequences was performed using the Geneious software, version 9.0.5 (Kearse et al., 2012). Alignment and identity matrix analyses were conducted using MAFFT (Katoh \& Standley, 2013) and BioEdit (Hall, 1999). Sequences generated in this study were submitted to the GenBank NCBI (National Center for Biotechnology Information) database (http://www.ncbi.nlm.nih.gov/) and assigned accession numbers shown in Table 1. Reference sequences of NDV genotypes (Diel et al., 2012; Snoeck et al., 2013) required for further analyses were retrieved from the GenBank database. Phylogenetic analyses were performed and trees were constructed using the IQ-tree software version 1.1.3 (Minh et al., 2013; Nguyen et al., 2014) based on maximum likelihood analysis of phylogenetic relationship after selection of the best fit substitution model. Finally, trees were viewed and edited using FigTree v1.4.2 software (http://tree.bio.ed.ac.uk/software/figtree/) and Inkscape 0.51 .

\section{Full Genome Sequencing}

Full genome sequences of four successfully isolated NDV strains, namely, R1954-11-cl-1, R1973-11-cl-2, R1973-11-cl-4 and AR2178-14, representing three genotypes previously reported in Egypt, were carried out. Briefly, RNA was purified from isolates using TRIzol LS reagent (LifeTechnologies, Darmstadt, Germany) and an RNeasy mini kit (Qiagen, Hilden, Germany) with on-column DNase digestion according to the manufacturer's instructions. Conversion of RNA into double-stranded DNA was performed using a cDNA synthesis system (Roche, Mannheim, Germany). Library construction was done as previously described (Juozapaitis et 
al., 2014). Sequencing was performed on an Illumina MiSeq Instrument using the MiSeq reagent kit version 3 (Illumina, San Diego, CA, USA).

Assembly of the sequence data was done using the Genome Sequencer version 2.6 software suite (Roche), and NDV-related contigs were identified with BLASTn (BLASTn; http://blast.ncbi.nlm.nih.gov/Blast.cgi). The obtained full-length NDV sequences have been submitted to GenBank (Table 1). Further, the open reading frame (ORF) analysis and the annotation of the genome were carried out using the Geneious software.

Phylogenetic analyses were carried out for both of F-gene (partial/full) and the whole genome sequence separately using references viruses as mentioned in the previous section.

\section{Pathotyping}

Intracerebral pathogenicity index (ICPI) was determined following European guidelines (CEC, 1992). Ten one-day-old SPF chickens were inoculated intracerebrally with $0.05 \mathrm{ml}$ of $10^{-1}$ diluted virus stock $(\mathrm{HA}>32$ ) and examined daily for 8 days. The ICPI is calculated by scoring each bird every day from 0 (normal), 1 (sick) and to 2 (dead). Based on this score NDV-isolates are considered lentogenic (-0.7), mesogenic $(>0,7$ 1.3) or velogenic $(>1,5)$ (Alexander, 1998). Animal experiments were carried out in accordance with the legally approved protocol (MV-LALLF- /7221.3-1.1-030/13). Specific pathogen free White leghorn chickens purchased from Lohmann Animal Health, Cuxhaven, Germany, were raised in isolation units at FLI until age of inoculation. Infection and immunization experiments were performed in in separated cages at animal biosafety level-3 (BSL-3). Feed and water were provided daily.

Preparation of polyclonal anti-NDV chicken antisera

Four reference NDV strains, clone30, Ulster, R151/94, and R1468/12, representing the avian orthoavulavirus1, 2.II, 2.VI (PPMV-1) and 2.VII.1.1, respectively, were selected for preparation of polyclonal anti-NDV chicken antiserum in the FLI animal facility. Viruses were first inactivated by betapropiolactone (Sigma; $0,05 \%$,) treatment and $0.5 \mathrm{~mL}$ of each virus was injected in four 4-week-old-SPF White Leghorn chickens via subcutaneous route after mixing with Freund's complete adjuvant (1:1). A second immunization shot was given after 4 weeks. Collection of serum was performed at 7 days after the booster immunization, followed by inactivation at $56^{\circ} \mathrm{C}$ for $30 \mathrm{~min}$ and stored at $-20{ }^{\circ} \mathrm{C}$ until further use. Immunizations were carried out in accordance with the legally approved protocol (MV-LALLF- 7221.3-2.5-010/10).

\section{Antigenic characterization}

Antigenic analysis of three Egyptian NDVs AR2178-14, R1973-11-cl4 and R1954-11-cl6 as well as of another five reference strains was performed by hemagglutination inhibition (HI) assay, as described (CEC, 1992). In addition to a panel of four polyclonal sera (see previous section) and five HN-specific monoclonal antibodies were used (mAb 617/161, mAb U85, mAb 7D4 were a generous gift from AVLA-Weybridge and mAb 39-2, mAb 10 were a gift from Bernd Köllner, FLI)

\section{Results}

$R T-q P C R$, virus isolation and purification

Molecular pathotyping based on RT-qPCR using the primers and probes specific for avirulent and virulent pathotypes (RT-F-qPCR) revealed that two Egyptian NDV isolates of the year 2011 were positive for lentogenic as well as virulent pathotypes, indicating mixed infection. The consensus sequence of the proteolytic cleavage site within the $\mathrm{F}_{2}$-protein of one mixed isolate (R1954-11) represents a polybasic recognition motive, characteristic for virulent meso-/velogenic pathotypes (. This would be in agreement with the obtained ICPI of 0.91 , representing a mesogenic pathotype. In contrast, the sequence of the second mixed isolate R1973/11 represents a cleavage site of lentogenic vaccine type viruses but had an ICPI of 1.88, clearly identifying the isolate as velogenic. By plaque cloning, clones with mono- (R1954/-11- cl1; R1973/11-cl 2) or polybasic cleavage sites (R1954/11-cl 6; R1973/11-cl 4) could be obtained from both samples. For these purified clones, unambiguous reactivity patterns in pathotype-specific RT-qPCRs were consistent with their sequences of 
the proteolytic cleavage site and their ICPI values even though the ICPI value of R1973-11-cl 2 was slightly above the threshold of 0.7 between lentogenic to mesogenic strains.

\section{Genetic and phylogenetic characterization}

Sequences of the F2-fragment, including the proteolytic cleavage site were used for initial phylogenic characterization (358 nt). BLAST tools indicated highest homology for isolate AR2178/2014, with Egyptian Avian avulavirus-1/168-2012 (Genbank accession: MN381174) with nt and aa identities of $99 \%$ and $99 \%$, respectively, clustering in phylogenetic analysis with genotype 2.VII.1.1 viruses (supplementary Fig 1.). In contrast, virulent NDV clones from 2011 (R1954/2011-cl 6; R1973/2011-cl 4) showed highest identities to EG-14/90 (Genbank accession: DQ096604) and chicken/Nigeria/2006 (Genbank accession: MH092825) respectively The lentogenic clone R1954/2011-cl 1 matched $100 \%$ with vaccine strain LaSota (Genbank accession: AF077761), while R1973/2011-cl 2 matched with vaccine strain Hitchner B1 (Genbank accession: JN872151). When sequence collection of the genotyping group (Dimitrov et al. 2019) were used to create a first phylogenetic analysis (S1), viruses grouped to genotype 2.VII.1.1 (AR2178/2014), 2.XXI (R1954/2011cl 6; R1973/2011-cl 4) and 2.II (R1954/2011-cl 1; R1973/2011-cl 2). While genotype 2.VII.1.1 viruses are circulating in Egypt from 2012 up to 2019 (Fig. S1), no further sequences for NDV genotype 2.XXI have been reported for chickens after 2011. However subsequent viruses from genotype 2.XXI belong to sub-genotype 2. XXI.1.1 and are derived from pigeons.

\section{Full genome sequence analysis}

To verify and further analyze selected NDV isolates, full genome sequences were generated for both vaccine type clones (R1954/2011-cl 1, R1973/2011-cl 2) as well as for genotype 2.XXI (R1973/2011-cl 4) and 2.VII.1.1 (AR2178/2014) isolates. The genomes obtained for the lentogenic NDV clones R1954/2011-cl 1 and R1973/2011-cl 2 were 15.135 and 15.150 nt long, whereas the genomes of the virulent strains AR2178/2014 and clone R1973/2011-cl 4 were 15,179 and 15,165 nt long with a 6-nt-insertion ACCCCC or CCTCTC respectively in the untranslated region (UTR) downstream of the NP gene.

The two NDV strains of R1954/2011-cl 1 and R1973/2011-cl 2 showed 99\% nt similarity with the NDV strain LaSota C5 (accession: KC844235) (Table 2). On the other hand, the velogenic NDV strain AR2178/2014 showed the highest nt sequence similarity of 99\% to the Chinese NDV strain SD04/2011 (accession: JQ015296), whereas strain R1973/2011-cl 4 showed $94 \%$ nt similarity with the Japanese NDV strain Osaka/2440/1969 (accession: AB853926).

Phylogenic analysis of the full $\mathrm{F}$ gene (Fig. 1) as well as the whole genome sequence (Fig. 2) confirmed the phylogrouping obtained by partial F-gene analysis (Fig. S1). Genome organization of the four fully sequenced NDV strains was found to be identical to other Avian orthoavulavirus -1, containing six genes in the order of 3'-NP-P-M-F-HN-L-5', encoding at least seven proteins; N, P, V, M, F, HN and L. Analysis of the F protein of the four NDV strains revealed that three strains, namely, R1954/2011-cl 1, R1973/2011-cl 2 and AR2178/2014 (genotype 2.II, and 2.VII.11, respectively) possessed six potential N-glycosylation sites (NGS); five within the ectodomain at amino acid (aa) positions 85, 191, 366, 447, and 471 and one in the cytoplasmic domain at position 542. Strain R1973/2011-cl 4 (genotype 2.XXI) possessed the same five ectodomain NGS and an additional one at position aa 4 (https://prosite.expasy.org/PDOC00001 ).

The HN proteins of the two NDV strains R1973/2011-cl 4 and AR2178/2014 (genotype 2.XXI and 2.VII.1.1, respectively) were 571 aa long, which is 6 aa shorter than those of the NDV strains of genotype 2.II, namely, R1954/2011-cl 1 and R1973/2011-cl 4 (577 aa). The HN protein of all genotypes possessed five potential NGS. Four NGS at positions 119, 341, 433 and 481 were common in the four NDV strains, whereas NDV strains R1973/2011-cl 4 and AR2178/2014 (genotype 2.XXI and 2.VIIb, respectively) possessed an additional NGS at site 508, and strains R1954/2011-cl 1 and R1973-11-cl 2 (genotype 2.II) at position 538.

Comparing the level of homology of the different isolates to the La Sota vaccine strain (Accession No. AF077761) confirms the close relation in between the genotype 2.II viruses. Regarding genotype 2.XXI and 2.VII, both genotypes accumulated mutations compared to La Sota vaccine strain, but these changes were 
present for both viruses to a comparable level with homology of 81.0 to $85.5 \%$. For the different genes, no striking differences are evident. Comparing the amino acid (aa) composition of genotype 2.XXI and 2.VII.1.1, the homology to 2.II strain La Sota is higher than on the level of nucleotides, with 92 to $87.9 \%$ for NP, M, F, HN and L-protein. It is striking that the homology of the P-protein was considerably lower than the other genes with 80.1 and $81.9 \%$ for genotype 2.XXI and 2.VII.1.1 respectively. When focusing on the HN protein, responsible for receptor mediated binding, our data reveal overall aa differences of $11,7 \%$ and $11,9 \%$ for 2.XXI and 2.VII.1.1 viruses respectively compared to LaSota vaccine type (Table.3). Mutations accumulated within the protein fragment encompassing the tail, transmembrane part and stalk region (aa 1-125; 22,4\% and 25,6\%), whereas the globular head (aa 126-570), bearing relevant antigenic sites, was underrepresented and had an aa divergence of only $8,8 \%$ and $8,1 \%$ for genotypes 2.XXI and 2.VII.1.1 respectively. Among the aa in the globular head, 21 of 39 and 35 substitutions were identical for both 2.XXI and 2.VII.1.1 viruses. These changes include 6 single point mutations in sites that were described to be part of neutralization sensitive epitopes (Y203H, N263K, N347K, E494D, G495E and V514E) of the HN-protein (Table 3), i.e. site site 23; site 3, site I, site12, site II, site 2 (Iorio et al., 1989; Iorio et al., 1991) (Iorio). In an additional six positions both viruses had changes compared to LaSota (aa, 266, 288, 310, 433, 443, 509) but differed in aa composition. Unique changes were evident on 9 and 10 positions for genotype 2.XXI and 2.VII.1.1 viruses respectively. None of those isolate-specific sites affected known functional sites directly. For the vaccine type isolates only R1973/2011-cl 1 had 5 alterations, that were all located within the globular head of the HN-protein, with mutations in epitope $23(\mathrm{Y} 203 \mathrm{H})$ and NA binding site (A502V) that were identical for the genotype 2.XXI and 2.VII.1.1 viruses.

\section{Antigenic characterization}

By cross hemagglutination inhibition (HI) assays (Table 4) reaction patterns of sera directed against clone 30 (2.II) or Ulster (2.I.2) and the Egyptian isolates of genotype 2.II were indistinguishable. Reactivity even with a phylogenetically distant class 1 APMV-1 isolate (R2919/06) was in the range of $2 \log _{2}$ steps. In contrast, reactivity of a serum raised against another genotype 2.VII.1.1 virus (R1468/12) was considerably lower with genotype 2.II and 2.I viruses $(3 \log 2)$ as well as with the class 1 virus $(4 \log 2)$. However, genotype 2.VII.1.1 specific serum did not distinguish between Egyptian genotype 2.VI and 2.VII.1.1 viruses. In addition, a serum raised against classical PPMV-1 virus (R151/94) assigned to genotype 2.VI did show equal reactivity to both genotype 2.XXI chicken isolates, but reactivity was decreased to genotype 2.II and 2.I and class 1 virus and to a lesser extent to genotype 2.VII.1.1 viruses. Reactivity profiles obtained by HI with a panel of mAbs, further highlighted antigenic differences between PPMV-1 and other genotype 2.XXI strains (R1973/2011-cl 4 and R1954/2011-cl 6). A second set of mAbs, reactive with vaccine type genotype 2.II viruses varied in their reactivity profile: whereas mAb U85 recognized genotype 2.II, 2.I and R1468/12 (2.VII.1.1) viruses, $\mathrm{mAb} 7 \mathrm{D} 4$ was specific only for vaccine type 2.II viruses, and mAb 10 recognized an epitope present in genotype 2.II and 2.I viruses. These data strongly suggested, that epitope specific difference existed, but that they were of minor importance for recognition by polyclonal sera that comprise antibodies directed against multiple epitopes.

\section{Discussion}

NDV continues to cause outbreaks and substantial losses among the poultry industry worldwide (Brown \& Bevins, 2017; Dimitrov et al., 2016). In Egypt, in spite of the routine implementation of ND vaccination, detection of NDV of genotypes 2.II, 2.VI, and 2.VII.1.1 have been reported continuously over the last 10 years (Orabi et al., 2017; Saad et al., 2017; Sabra et al., 2017). Continuously growing phylogenic diversity points to the evolution of virus populations that display a genetic distance to vaccine type NDV genotype 2.II. This observation has been taken as indication for antigenic vaccine mismatch and a suspected cause for vaccine failure (Dimitrov et al., 2017; Nagy et al., 2020). In this model new NDV strains emerge because of an change in antigenic sites relevant for protection. To test this hypothesis an "old" NDV isolate from 2011 representing NDV genotype 2.XXI was compared to a currently dominating NDV genotype in chickens in Egypt (2.VII.1.), with specific focus on sites blocking receptor binding and sensitive for neutralization.

Based on the new classification system proposed by Dimitrov et al. (2019), viruses sequenced in the current 
study were grouped phylogenetically as genotype 2.II, 2.XXI.1.1 and 2.VII.1.1. The latter originated from China and circulated extensively in the Middle East (Ewies et al., 2017; Radwan et al., 2013; Saad et al., 2017). In contrast NDV genotype 2.XXI has not been recorded in poultry for almost the last decade. This group of viruses are descendent of genotype 2.XX and together with the new genotype 2.VI form a monophyletic branch (Dimitrov et al., 2019)(Dimitrov et al. 2019). Evolutionary time scale analysis indicate that genotype 2.XX appeared around in 1959 in poultry (Chong et al., 2013) forming sub-lineage 2.VIai that arose in Eastern Asia and later circulated in Europe, causing sporadic ND outbreaks in Western Europe and Bulgaria in the mid 1990s (Ujvari et al., 2003) (Czegledi et al. 2002, Ujvari et al. 2003). In the second subgroup 2.VIaii (Chong et al., 2013) that is now consolidated in the new genotype 2.XXI, repeated chicken-to-pigeon transmissions were observed that eventually was successful to establish pandemic PPMV-1 infection, and forming the new genotype 2.VI (former genotype 2.VIb) (Chong et al., 2013; Ujvari et al., 2003). In addition, other descendants of genotype 2.XXI established infections in the pigeon population. These pigeon derived viruses are accumulated in three sub-genotypes, i.e. 2.XXI.1.1; 2.XXI.1.2 and 2.XXI.2 respectively with genotype 2.XXI.1.1 subsequently detected in Egypt (Sabra et al. (2017). These phylogenetic data highlight the possible interspecies transmission of genotype 2.XXI viruses but clearly support the notion that isolates R1954/11-cl 1 R1973/11-cl 2 are original poultry derived strains and not a spill-over infection from pigeon to poultry as described for PPMV-1 in the 1980s in England (Alexander et al., 1985). This notion of genotype 2.XXI being a virus circulating in poultry is further supported by the finding, that closely related viruses were detected in chickens in Ethiopia in 2011 and 2012 (De Almeida et al., 2013). However, subsequent outbreaks in Egypt are dominated by genotype 2.VII.1.1 viruses, indicating that genotype 2.XXI was superseded. It was considered that ND vaccination is a driving force for virus evolution (Chong et al., 2010) with selection of escape variants (Cho et al., 2007; Cho et al., 2008). In this respect genotype 2.VII.1.1. virus (AR 2178/2014) should harbor mutations within neutralizing sites not present in genotype 2.XXI virus (R1973/11-cl 2). However, when analyzing sites that are part of neutralizing epitopes, all 6 mutations present in genotype 2.VII.1.1. virus were also present in genotype 2.XXI virus. It was remarkable, that for 5 out 6 sites, mutations were associated with a change of principle properties of the amino acid, i.e. change from hydrophobic to alkaline, positively charged (Y203H), from acidic, negatively charged to alkaline, positive charged (N263K, E347K), from neutral to acidic, negatively charged (G494D) or from hydrophobic to acidic, negatively charged (V495E). That indicates, that mutations might be associated with a change of antigenic properties. In line with this assumption are the data from the cross HI-tests. Reactivity profile with $\mathrm{mAb}$ revealed changes in specific epitopes and also polyclonal sera discriminated between viruses from different genotypes. However, overall our data support the notion that NDV / avian orthoavulavirus -1 is still a single serogroup (Miller \& Koch, 2013) and a discrimination between genotype 2.XX and 2.VII was not evident. Taking together our data do not indicate that a switch of antigenic sites were the driving selection criterion for the replacement of genotype 2.XXI by genotype 2.VII.1.1. This would be in line with the observation that the overall mutation rate of NDV indicates strong purifying (negative) selection for all proteins (Chong et al., 2013; Miller et al., 2009).

It was striking that both historical 2.XXI isolates contained also a vaccine type virus (genotype 2.II). Coinfections were revealed initially by RT-qPCR but would have been concealed by regular pathotyping using Sanger-sequencing: The $\mathrm{F}$ protein cleavage site of virulent strains carries a polybasic motive ( ${ }^{112} \mathrm{R} / \mathrm{K}-\mathrm{R}-\mathrm{Q}-$ $\mathrm{K} / \mathrm{R}-\mathrm{R}^{*} \mathrm{~F}^{117}$ ) and avirulent NDV strains harbor ${ }^{112} \mathrm{G} / \mathrm{E}-\mathrm{K} / \mathrm{R}-\mathrm{Q}-\mathrm{G} / \mathrm{E}-\mathrm{R}^{*} \mathrm{~L}^{116}$ and a leucine at position 116 at the F protein cleavage site (Collins et al., 1993; de Leeuw et al., 2005; Peeters et al., 1999; Romer-Oberdorfer et al., 1999).

For isolate R1954/11 the obtained polybasic cleavage site corresponded to the ICPI, however pointing to a mesogenic pathotype. Sanger-sequence of isolate R1973/11 pointed to avirulent pathotype, but the ICPI result clearly revealed a velogenic pathotype. Such apparently contradicting results have been published before (Nagy et al., 2020; Tan et al., 2008), but were not further resolved. After observing such contradicting results, we plaque-cloned both isolates and were able to separate two different NDV: beside genotype 2.XXI virus, a vaccine type virus (genotype 2.II) was obtained. Subsequent pathotyping of the cloned viruses by ICPI now matched with the sequence of the proteolytic cleavage site and full genome sequence comparison of 
the sequenced lentogenic strains reveal a close relation to vaccine strain Hitchner B1 / JN872151 (R1973/11cl 2) or LaSota /AF077761 (R1954/11-cl 1). This finding further supports the notion of re-isolated vaccine strains and is clearly distinct from described virulent genotype 2.II viruses, detected in ND outbreaks in 2006 in Egypt (Mohamed et al., 2011). All viruses from the year 2006 (NDV/chicken/Egypt/2-4/2006; FJ969393, FJ969394, FJ969395) belong to genotype 2.II but are of velogenic pathotype with ICPI values between 1,6 to 1.8 and a have corresponding proteolytic polybasic cleavage site (RRQKR*FIG). The re-isolation of vaccine type virus in line with earlier description (Abolnik et al., 2004; Nagy et al., 2020) and it is conceivable, that emergency vaccination in flocks with suspected ND might lead to such double infections. Those circumstances have been considered by the OIE (OIE, 2018) when defining criteria for virulence: The definition includes the sequence information of the proteolytic cleavage site, but has the amendment "Failure to demonstrate the characteristic pattern of amino acid residues as described above would require characterization of the isolated virus by an ICPI test". In the brain multicycle replication is restricted to virulent NDV due to the cleavability of the F-protein (Nagai et al., 1976; Rott, 1979) and thus brain passage lead to enrichment of virulent NDV. In case of contradicting results as described by others (Nagy et al., 2020; Tan et al., 2008) sequencing of re-isolated virus from the brain is indicated.

The failure to demonstrate antigenic escape in genotype 2.XXI precursor and its genotype 2.VII.1.1 successor virus indicate that other factors, maybe founded in regulatory viral genes, contribute to the assertiveness of a specific virus population. Co-infections of vaccine type and wild type NDV point to a conjecturable practice of emergency vaccination and unrestricted handling of diseased poultry, thereby fostering ND endemicity in Egypt. Apart from moecular epidemiologic studies on NDV transmission dynamics combatting ND endemicity in Egypt certainly requires improved herd and trading management.

\section{Declarations}

\section{Competing interests}

The authors declare that they have no competing interests.

Acknowledgment

The authors thank Cornelia Illing, FLI, Germany, for excellent technical support. We are grateful to colleagues and co-workers at NLQP and Beni-Suef university, Egypt, for helping in field samples collection.

\section{References}

Abolnik, C., Horner, R. F., Bisschop, S. P., Parker, M. E., Romito, M., \& Viljoen, G. J. (2004). A phylogenetic study of South African Newcastle disease virus strains isolated between 1990 and 2002 suggests epidemiological origins in the Far East. Arch Virol, 149 (3), 603-619. doi:10.1007/s00705-003-0218-2

Aldous, E. W., Collins, M. S., McGoldrick, A., \& Alexander, D. J. (2001). Rapid pathotyping of Newcastle disease virus (NDV) using fluorogenic probes in a PCR assay. Vet Microbiol, 80 (3), 201-212.

Aldous, E. W., Mynn, J. K., Banks, J., \& Alexander, D. J. (2003). A molecular epidemiological study of avian paramyxovirus type 1 (Newcastle disease virus) isolates by phylogenetic analysis of a partial nucleotide sequence of the fusion protein gene.Avian Pathol, 32 (3), 239-256. doi:10.1080/030794503100009783

Alexander. (1998). Newcastle disease and other avian paramyxoviruses. A laboratory manual for the isolation and identification of avian pathogens. American Association of Avian Pathologists, Kennett: Square. , 156-163.

Alexander. (2000). Newcastle disease and other avian paramyxoviruses. Rev Sci Tech, 19 (2), 443-462.

Alexander, D. J., Russell, P. H., Parsons, G., Elzein, E. M. E. A., Ballouh, A., Cernik, K., . . . Tumova, B. (1985). Antigenic and biological characterisation of avian paramyxovirus type I isolates from pigeons - an international collaborative study. Avian Pathology, 14 (3), 365-376. doi:10.1080/03079458508436238 
Brown, V. R., \& Bevins, S. N. (2017). Correction to: A review of virulent Newcastle disease viruses in the United States and the role of wild birds in viral persistence and spread. Vet Res, 48 (1), 77. doi:10.1186/s13567-017-0485-7

CEC. (1992). Council Directive 92/66/EEC introducing Community measures for the control of Newcastle disease. . Official Journal of the European Community., L 260:1-20.

Chong, Y. L., Lam, T. T., Kim, O., Lu, H., Dunn, P., \& Poss, M. (2013). Successful establishment and global dispersal of genotype VI avian paramyxovirus serotype 1 after cross species transmission. Infect Genet Evol, 17 , 260-268. doi:10.1016/j.meegid.2013.04.025

Chong, Y. L., Padhi, A., Hudson, P. J., \& Poss, M. (2010). The effect of vaccination on the evolution and population dynamics of avian paramyxovirus-1. PLoS Pathog, 6 (4), e1000872. doi:10.1371/journal.ppat.1000872

Collins, M. S., Bashiruddin, J. B., \& Alexander, D. J. (1993). Deduced amino acid sequences at the fusion protein cleavage site of Newcastle disease viruses showing variation in antigenicity and pathogenicity. Arch Virol, 128 (3), 363-370. doi:10.1007/bf01309446

Czegledi, A., Herczeg, J., Hadjiev, G., Doumanova, L., Wehmann, E., \& Lomniczi, B. (2002). The occurrence of five major Newcastle disease virus genotypes (II, IV, V, VI and VIIb) in Bulgaria between 1959 and 1996. Epidemiol Infect, 129 (3), 679-688.

Czegledi, A., Wehmann, E., \& Lomniczi, B. (2003). On the origins and relationships of Newcastle disease virus vaccine strains Hertfordshire and Mukteswar, and virulent strain Herts'33. Avian Pathol, 32 (3), 271-276. doi:10.1080/0307945031000097868

Daubney, R., \& Mansy, W. (1948). The occurrence of Newcastle disease in Egypt. J Comp Pathol Ther, 58 (3), 189-200.

de Almeida, R. S., Hammoumi, S., Gil, P., Briand, F. X., Molia, S., Gaidet, N., . . . Albina, E. (2013). New avian paramyxoviruses type I strains identified in Africa provide new outcomes for phylogeny reconstruction and genotype classification.PLoS One, 8 (10), e76413. doi:10.1371/journal.pone.0076413

de Leeuw, O. S., Koch, G., Hartog, L., Ravenshorst, N., \& Peeters, B. P. (2005). Virulence of Newcastle disease virus is determined by the cleavage site of the fusion protein and by both the stem region and globular head of the haemagglutinin-neuraminidase protein. J Gen Virol, 86 (Pt 6), 1759-1769. doi:10.1099/vir.0.80822-0

Diel, D. G., da Silva, L. H., Liu, H., Wang, Z., Miller, P. J., \& Afonso, C. L. (2012). Genetic diversity of avian paramyxovirus type 1: proposal for a unified nomenclature and classification system of Newcastle disease virus genotypes. Infect Genet Evol, 12 (8), 1770-1779. doi:10.1016/j.meegid.2012.07.012

Dimitrov, K. M., Abolnik, C., Afonso, C. L., Albina, E., Bahl, J., Berg, M., . . . Wong, F. Y. K. (2019). Updated unified phylogenetic classification system and revised nomenclature for Newcastle disease virus. Infect Genet Evol, 74 , 103917. doi:10.1016/j.meegid.2019.103917

Dimitrov, K. M., Afonso, C. L., Yu, Q., \& Miller, P. J. (2017). Newcastle disease vaccines-A solved problem or a continuous challenge? Vet Microbiol, 206 , 126-136. doi:10.1016/j.vetmic.2016.12.019

Dimitrov, K. M., Lee, D. H., Williams-Coplin, D., Olivier, T. L., Miller, P. J., \& Afonso, C. L. (2016). Newcastle Disease Viruses Causing Recent Outbreaks Worldwide Show Unexpectedly High Genetic Similarity to Historical Virulent Isolates from the 1940s. J Clin Microbiol, 54 (5), 1228-1235. doi:10.1128/jcm.03044-15

Ewies, S. S., Ali, A., Tamam, S. M., \& Madbouly, H. M. (2017). Molecular characterization of Newcastle disease virus (genotype VII) from broiler chickens in Egypt.Beni-Suef University Journal of Basic and Applied Sciences, 6 (3), 232-237. doi:https://doi.org/10.1016/j.bjbas.2017.04.004 
Grund, C., Abdelwhab el, S. M., Arafa, A. S., Ziller, M., Hassan, M. K., Aly, M. M., . . . Beer, M. (2011). Highly pathogenic avian influenza virus H5N1 from Egypt escapes vaccine-induced immunity but confers clinical protection against a heterologous clade 2.2.1 Egyptian isolate. Vaccine, 29 (33), 5567-5573. doi:10.1016/j.vaccine.2011.01.006

Hall, T. (1999). BioEdit: a user-friendly biological sequence alignment editor and analysis program for Windows 95/98/NT. Nucleic Acids Symp. Ser., 41 , 95-98.

Herczeg, J., Pascucci, S., Massi, P., Luini, M., Selli, L., Capua, I., \& Lomniczi, B. (2001). A longitudinal study of velogenic Newcastle disease virus genotypes isolated in Italy between 1960 and 2000. Avian Pathol, 30 (2), 163-168. doi:10.1080/03079450120044000

Herczeg, J., Wehmann, E., Bragg, R. R., Travassos Dias, P. M., Hadjiev, G., Werner, O., \& Lomniczi, B. (1999). Two novel genetic groups (VIIb and VIII) responsible for recent Newcastle disease outbreaks in Southern Africa, one (VIIb) of which reached Southern Europe. Arch Virol, 144 (11), 2087-2099.

Iorio, R. M., Glickman, R. L., Riel, A. M., Sheehan, J. P., \& Bratt, M. A. (1989). Functional and neutralization profile of seven overlapping antigenic sites on the HN glycoprotein of Newcastle disease virus: monoclonal antibodies to some sites prevent viral attachment. Virus Res, 13 (3), 245-261. doi:10.1016/01681702(89)90019-1

Iorio, R. M., Syddall, R. J., Sheehan, J. P., Bratt, M. A., Glickman, R. L., \& Riel, A. M. (1991). Neutralization map of the hemagglutinin-neuraminidase glycoprotein of Newcastle disease virus: domains recognized by monoclonal antibodies that prevent receptor recognition. J Virol, 65 (9), 4999-5006. doi:10.1128/JVI.65.9.4999-5006.1991

Juozapaitis, M., Aguiar Moreira, E., Mena, I., Giese, S., Riegger, D., Pohlmann, A., . . . Schwemmle, M. (2014). An infectious bat-derived chimeric influenza virus harbouring the entry machinery of an influenza A virus. Nat Commun, 5 , 4448. doi:10.1038/ncomms5448

Katoh, K., \& Standley, D. M. (2013). MAFFT multiple sequence alignment software version 7: improvements in performance and usability. Mol Biol Evol, 30 (4), 772-780. doi:10.1093/molbev/mst010

Kawaguchi, T., Nomura, K., Hirayama, Y., \& Kitagawa, T. (1987). Establishment and characterization of a chicken hepatocellular carcinoma cell line, LMH. Cancer Res, 47 (16), 4460-4464.

Kearse, M., Moir, R., Wilson, A., Stones-Havas, S., Cheung, M., Sturrock, S., . . . Drummond, A. (2012). Geneious Basic: an integrated and extendable desktop software platform for the organization and analysis of sequence data.Bioinformatics, 28 (12), 1647-1649. doi:10.1093/bioinformatics/bts199

Kim, L. M., King, D. J., Curry, P. E., Suarez, D. L., Swayne, D. E., Stallknecht, D. E., . . . Afonso, C. L. (2007). Phylogenetic diversity among low-virulence newcastle disease viruses from waterfowl and shorebirds and comparison of genotype distributions to those of poultry-origin isolates. J Virol, 81 (22), 12641-12653. doi:10.1128/jvi.00843-07

Lamb, R. A., \& Parks, G. D. (2007). Paramyxoviridae: the viruses and their replication. Fields virology, 5th ed. Wolters Kluwer-Lippincott Williams \&3 Wilkins, Philadelphia, PA. , 1449-1496. doi:10.1039/b611613m

Miller, P. J., Decanini, E. L., \& Afonso, C. L. (2010). Newcastle disease: evolution of genotypes and the related diagnostic challenges. Infect Genet Evol, 10 (1), 26-35. doi:10.1016/j.meegid.2009.09.012

Miller, P. J., Kim, L. M., Ip, H. S., \& Afonso, C. L. (2009). Evolutionary dynamics of Newcastle disease virus. Virology, 391 (1), 64-72. doi:10.1016/j.virol.2009.05.033

Miller, P. J., \& Koch, G. (2013). Newcastle disease, In: Swayne, D.E., Glisson, J.R., McDougald, L.R., Nolan, L.K., Suarez, D.L., Nair, V. (Eds.) Diseases of Poultry (13th ed.) Wiley-Blackwell, Hoboken, 89138. 
Minh, B. Q., Nguyen, M. A., \& von Haeseler, A. (2013). Ultrafast approximation for phylogenetic bootstrap. Mol Biol Evol, 30 (5), 1188-1195. doi:10.1093/molbev/mst024

Mohamed, M. H., Kumar, S., Paldurai, A., \& Samal, S. K. (2011). Sequence analysis of fusion protein gene of Newcastle disease virus isolated from outbreaks in Egypt during 2006. Virol J, 8, 237. doi:10.1186/1743$422 \mathrm{x}-8-237$

Moharam, I., Razik, A. A. E., Sultan, H., Ghezlan, M., Meseko, C., Franzke, K., . . . Grund, C. (2019). Investigation of suspected Newcastle disease (ND) outbreaks in Egypt uncovers a high virus velogenic ND virus burden in small-scale holdings and the presence of multiple pathogens. Avian Pathol, 48 (5), 406-415. doi:10.1080/03079457.2019.1612852

Nagai, Y., Klenk, H. D., \& Rott, R. (1976). Proteolytic cleavage of the viral glycoproteins and its significance for the virulence of Newcastle disease virus. Virology, 72 (2), 494-508. doi:10.1016/0042-6822(76)90178-1

Naguib, M. M., El-Kady, M. F., Luschow, D., Hassan, K. E., Arafa, A. S., El-Zanaty, A., . . Harder, T. C. (2017). New real time and conventional RT-PCRs for updated molecular diagnosis of infectious bronchitis virus infection (IBV) in chickens in Egypt associated with frequent co-infections with avian influenza and Newcastle Disease viruses. J Virol Methods, 245 , 19-27. doi:10.1016/j.jviromet.2017.02.018

Nagy, A., Ali, A., Zain El-Abideen, M. A., Kilany, W., \& Elsayed, M. (2020). Characterization and genetic analysis of recent and emergent virulent newcastle disease viruses in Egypt. Transbound Emerg Dis . doi:10.1111/tbed.13543

Nguyen, L. T., Schmidt, H. A., von Haeseler, A., \& Minh, B. Q. (2014). IQ-TREE: A fast and effective stochastic algorithm for estimating maximum likelihood phylogenies. Mol Biol Evol . doi:10.1093/molbev/msu300

OIE. (2012). Newcastle disease (infection with Newcastle disease virus). OIE terrestrial manual, Chapter 2.3.14 .

OIE. (2018). Newcastle disease. Terrestrial Manual: Chapter 3.3.14. Available online https://www.oie.int/fileadmin/Home/eng/Health_standards/tahm/3.03.14_NEWCASTLE_DIS.pdf. Accessed on 24.10.2020.

Orabi, A., Hussein, A., Saleh, A. A., El-Magd, M. A., \& Munir, M. (2017). Evolutionary insights into the fusion protein of Newcastle disease virus isolated from vaccinated chickens in 2016 in Egypt. Arch Virol, 162 (10), 3069-3079. doi:10.1007/s00705-017-3483-1

Peeters, B. P., de Leeuw, O. S., Koch, G., \& Gielkens, A. L. (1999). Rescue of Newcastle disease virus from cloned cDNA: evidence that cleavability of the fusion protein is a major determinant for virulence. $J$ Virol, 73 (6), 5001-5009. doi:10.1128/jvi.73.6.5001-5009.1999

Radwan, M. M., Darwish, S. F., El-Sabagh, I. M., El-Sanousi, A. A., \& Shalaby, M. A. (2013). Isolation and molecular characterization of Newcastle disease virus genotypes II and VIId in Egypt between 2011 and 2012. Virus Genes, 47 (2), 311-316. doi:10.1007/s11262-013-0950-y

Rima, B., Balkema-Buschmann, A., Dundon, W. G., Duprex, P., Easton, A., Fouchier, R., . . . Ictv Report, C. (2019). ICTV Virus Taxonomy Profile: Paramyxoviridae. J Gen Virol, 100 (12), 1593-1594. doi:10.1099/jgv.0.001328

Romer-Oberdorfer, A., Mundt, E., Mebatsion, T., Buchholz, U. J., \& Mettenleiter, T. C. (1999). Generation of recombinant lentogenic Newcastle disease virus from cDNA.J Gen Virol, 80 ( Pt 11), 2987-2995. doi:10.1099/0022-1317-80-11-2987

Rott, R. (1979). Molecular basis of infectivity and pathogenicity of myxovirus. Arch Virol, 59 (4), 285-298. doi:10.1007/BF01317469 
Saad, A. M., Samy, A., Soliman, M. A., Arafa, A., Zanaty, A., Hassan, M. K., . . . Hussein, A. H. (2017). Genotypic and pathogenic characterization of genotype VII Newcastle disease viruses isolated from commercial farms in Egypt and evaluation of heterologous antibody responses. Arch Virol, 162 (7), 19851994. doi:10.1007/s00705-017-3336-y

Sabra, M., Dimitrov, K. M., Goraichuk, I. V., Wajid, A., Sharma, P., Williams-Coplin, D., . . . Afonso, C. L. (2017). Phylogenetic assessment reveals continuous evolution and circulation of pigeon-derived virulent avian avulaviruses 1 in Eastern Europe, Asia, and Africa. BMC Vet Res, 13 (1), 291. doi:10.1186/s12917017-1211-4

Samy, A., \& Naguib, M. M. (2018). Avian Respiratory Coinfection and Impact on Avian Influenza Pathogenicity in Domestic Poultry: Field and Experimental Findings.Vet Sci, 5 (1). doi:10.3390/vetsci5010023

Seal, B. S., King, D. J., \& Bennett, J. D. (1996). Characterization of Newcastle disease virus vaccines by biological properties and sequence analysis of the hemagglutinin-neuraminidase protein gene. Vaccine, 14 (8), 761-766. doi:10.1016/0264-410x(95)00252-v

Snoeck, C. J., Owoade, A. A., Couacy-Hymann, E., Alkali, B. R., Okwen, M. P., Adeyanju, A. T., . . . Muller, C. P. (2013). High genetic diversity of Newcastle disease virus in poultry in West and Central Africa: cocirculation of genotype XIV and newly defined genotypes XVII and XVIII. J Clin Microbiol, 51 (7), 2250-2260. doi:10.1128/JCM.00684-13

Steward, M., Vipond, I. B., Millar, N. S., \& Emmerson, P. T. (1993). RNA editing in Newcastle disease virus. J Gen Virol, 74 ( Pt 12) , 2539-2547. doi:10.1099/0022-1317-74-12-2539

Tan, L. T., Xu, H. Y., Wang, Y. L., Qin, Z. M., Sun, L., Liu, W. J., \& Cui, Z. Z. (2008). Molecular characterization of three new virulent Newcastle disease virus variants isolated in China. J Clin Microbiol, 46 (2), 750-753. doi:10.1128/jcm.01587-07

Ujvari, D., Wehmann, E., Kaleta, E. F., Werner, O., Savić, V., Nagy, E., . . . Lomniczi, B. (2003). Phylogenetic analysis reveals extensive evolution of avian paramyxovirus type 1 strains of pigeons (Columba livia) and suggests multiple species transmission. Virus Res, 96 (1-2), 63-73. doi:10.1016/s0168-1702(03)00173-4

Wehmann, E., Czegledi, A., Werner, O., Kaleta, E. F., \& Lomniczi, B. (2003). Occurrence of genotypes IV, V, VI and VIIa in Newcastle disease outbreaks in Germany between 1939 and 1995. Avian Pathol, 32 (2), 157-163. doi:10.1080/0307945021000071623

Wise, M. G., Suarez, D. L., Seal, B. S., Pedersen, J. C., Senne, D. A., King, D. J., . . . Spackman, E. (2004). Development of a real-time reverse-transcription PCR for detection of newcastle disease virus RNA in clinical samples. J Clin Microbiol, 42 (1), 329-338.

Table 1: Egyptian NDV isolates included in the study

\section{Hosted file}

image1.emf available at https://authorea.com/users/378915/articles/495316-genomic-andantigenic-properties-of-newcastle-disease-virus-genotypes-2-xx1-and-2-vii-from-egypt-donot-point-to-antigenic-drift-as-a-driving-force-of-spread

Table 2: Similarity comparisons of nucleotide and amino acid sequences of ND viruses sequenced in this study and the LaSota vaccine strain (AF077761).

\section{Hosted file}

image2.emf available at https://authorea.com/users/378915/articles/495316-genomic-andantigenic-properties-of-newcastle-disease-virus-genotypes-2-xx1-and-2-vii-from-egypt-donot-point-to-antigenic-drift-as-a-driving-force-of-spread 
Table 3: Amino acid mutations of Egyptian viruses within the HN-protein

\section{Hosted file}

image3.emf available at https://authorea.com/users/378915/articles/495316-genomic-andantigenic-properties-of-newcastle-disease-virus-genotypes-2-xx1-and-2-vii-from-egypt-donot-point-to-antigenic-drift-as-a-driving-force-of-spread

Table 4. Antigenic characterization of the NDV strains isolated in this study and NDV reference viruses as well as anti-F mAbs

\begin{tabular}{|c|c|c|c|c|c|c|}
\hline & \multicolumn{6}{|c|}{ Virus - Antigen } \\
\hline & 1 & $2 . I$ & 2.II & 2.VI & 2.XXI & 2.XXI \\
\hline Serum & $\mathrm{R} 2919 / 06$ & Ulster & Clone 30 & R $151 / 1994$ & R1954/2011-cl 6 & R1973/2011-cl \\
\hline a-Ulster (2.I) & 10 & 10 & 11 & 11 & 11 & 11 \\
\hline a-clone30 (2.II) & 6 & 7 & 8 & 7 & 8 & 9 \\
\hline a-R151/94 (PPMV-1) & 5 & 6 & 6 & 8 & 8 & 9 \\
\hline a-R1468/12 (2.VII) & 6 & 7 & 7 & 8 & 10 & 11 \\
\hline $\mathrm{mAb} 617 / 161$ & 3 & $<1$ & $<1$ & 11 & 1 & 2 \\
\hline mAb 39 & 3 & $<1$ & $<1$ & 6 & $<1$ & $<1$ \\
\hline mAb U85 & 4 & 8 & 11 & 1 & 2 & 4 \\
\hline mAb 7D4 & 3 & 1 & 11 & 1 & 1 & 2 \\
\hline mAb 10 & 1 & 5 & 8 & $<1$ & $<1$ & $<1$ \\
\hline HI to homologe virus & & & & {$[?] 2$} & {$[?] 2$} & {$[?] 4$} \\
\hline
\end{tabular}

Figure 1. Phylogenetic tree of ND viruses based on nucleotide sequence of full $\mathrm{F}$ gene.

Phylogenetic tree was based on maximum likelihood calculations (IQTree software) under the best fit model (model: GTR $+\mathrm{F}+\mathrm{I}+\mathrm{G} 4$ ) according to the Bayesian Information Criterion (BIC). Numbers at nodes represent measures of robustness based on an ultrafast bootstrap approach implemented in IQTree. The sequences of other reference strains as well as all publicly available Egyptian NDV were obtained from GenBank. Divisions and genotypes are designated according to Dimitrov et al., 2019. Egyptian viruses are colored in red, and black circle is infront of viruses of this study.

Figure 2. Phylogenetic tree of NDV viruses based on whole genome sequences.

Phylogenetic tree was based on maximum likelihood calculations (IQTree software) under the best fit model (model: GTR+F+I+G4) according to the Bayesian Information Criterion (BIC). Numbers at nodes represent measures of robustness based on an ultrafast bootstrap approach implemented in IQTree. The sequences of other reference strains as well as all publicly available Egyptian NDV were obtained from GenBank. Divisions and genotypes are designated according to Dimitrov et al., 2019. Egyptian viruses are colored in red, and black circle is in front of viruses of this study.

Figure S1. Phylogenetic tree of ND viruses based on partial sequence of the F gene (375 bp) spanning the cleavage site.

Phylogenetic tree was based on maximum likelihood calculations (IQTree software) under the best fit model (model: TIM2e+G4) according to the Bayesian Information Criterion (BIC). Numbers at nodes represent measures of robustness based on an ultrafast bootstrap approach implemented in IQTree. The sequences of other reference strains as well as all publicly available Egyptian NDV were obtained from GenBank. 


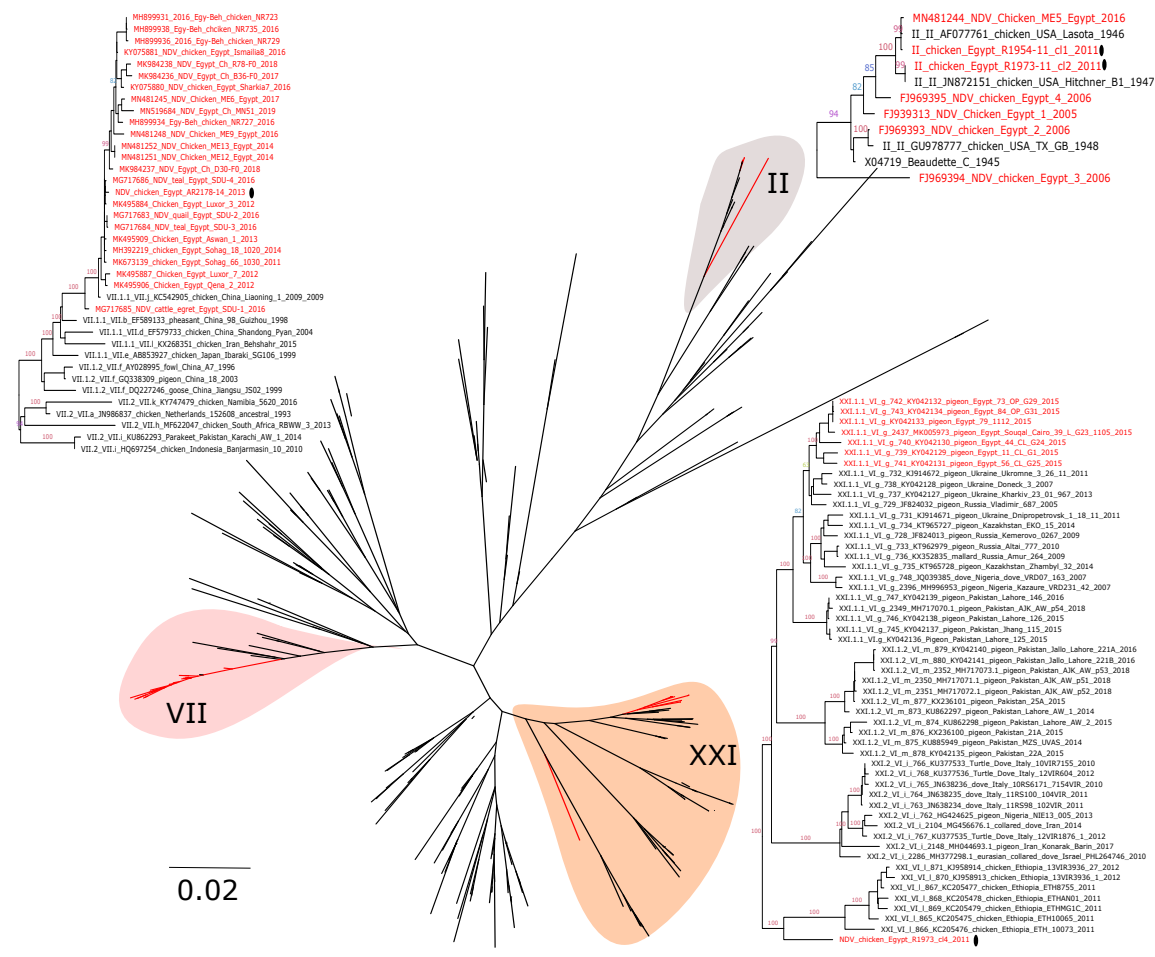




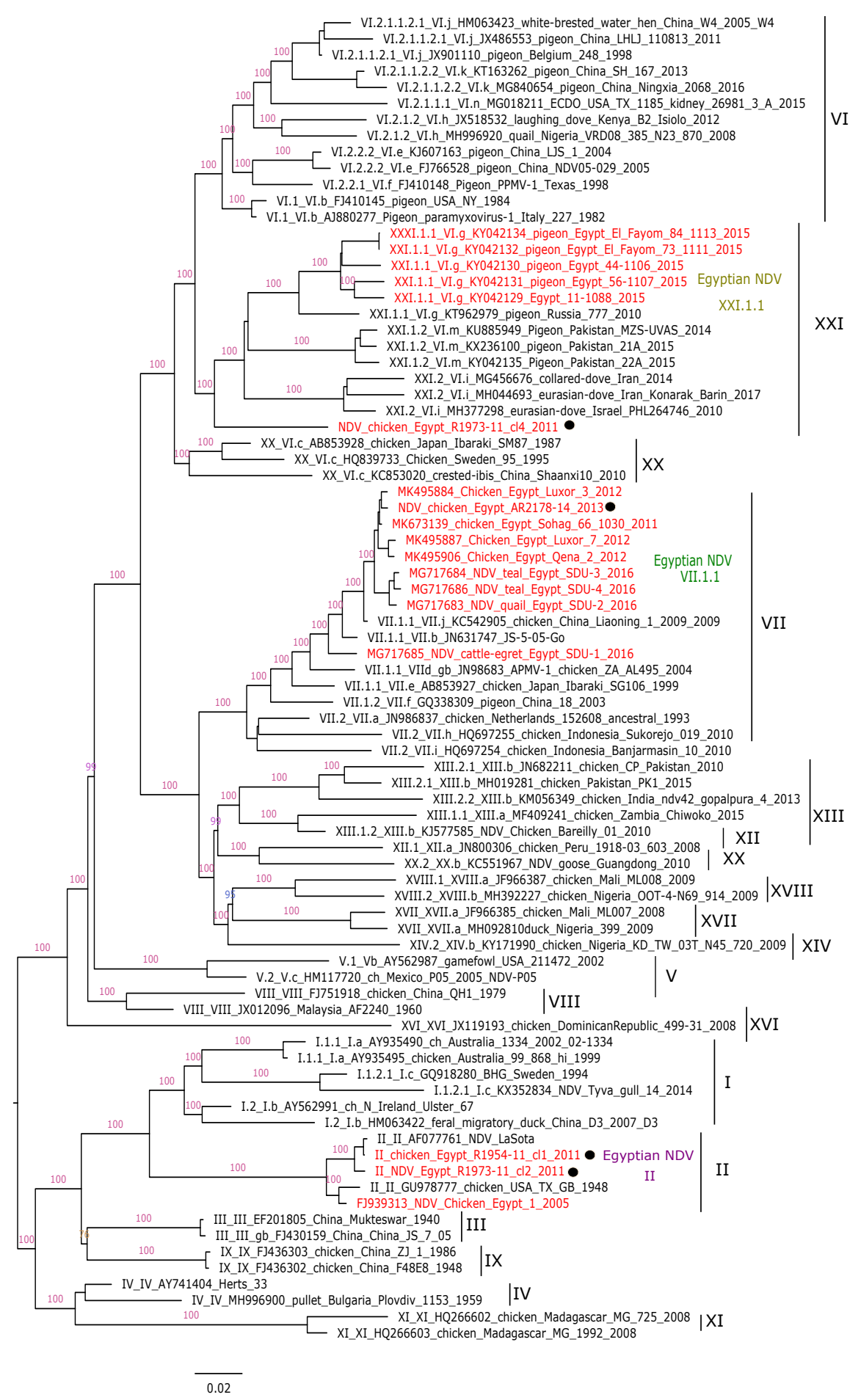

